\title{
Featured issue: Zinc Oxide
}

\author{
Arthur Willoughby
}

Published online: 16 December 2012

(c) Springer Science+Business Media New York 2012

In the first two issues of 2013 we are highlighting fields which are topics of particular current interest in our discipline, as we did in the first two issues of 2012. The February featured papers will be devoted to electronic materials related to Solar Energy Generation and Energy Storage, but in this (January) issue we again highlight the material featured in the February 2012 issue-Zinc Oxide. Reasons for the high interest in this material was outlined in my editorial in the February 2012 issues as follows.

While this II-VI semiconductor has been known for many years, the modern appreciation of its potential began in the 1950s, utilising properties such as piezoelectric, luminescent, and UV absorption characteristics, as well as its semiconductor features. The growth of single crystals facilitated the development of devices such as piezoelectric transducers, strain gauges, and pressure sensors, in addition to earlier devices such as varistors.

Further work on the growth of large single crystals was stimulated by the potential for lattice matched epitaxial growth of optoelectronic III-V nitrides, but more recently it has become apparent that $\mathrm{ZnO}$ might be a good optoelectronic material on its own account, owing to similarities with GaN. Having a similarly wide band-gap, and similar lattice dimensions, the key advantageous feature of $\mathrm{ZnO}$ is that it has a free exciton binding energy of $60 \mathrm{meV}$, more than twice that of GaN. This combination offers the possibility of UV laser action at room temperature, and has given rise to much research to address outstanding problems, including that on doping and thin film growth by several different growth techniques. Further work is also pursuing the potential of $\mathrm{ZnO}$-based dilute magnetic semiconductors in spintronics'.

Continued interest in this subject has led to this second Featured issue from papers submitted as regular submissions, and updates the position last year. We hope you will find this issue of interest, and that it will help to guide further work on this important topic.
A. Willoughby $(\square)$

Electronic Materials Research Group, University

of Southampton, Southampton, UK

e-mail: A.F.Willoughby@soton.ac.uk 